\title{
Demetra Application: An integrated genotype analysis web server for clinical genomics in endometriosis
}

\author{
LOUIS PAPAGEORGIOU ${ }^{1}$, MARIA I. ZERVOU ${ }^{2}$, DIMITRIOS VLACHAKIS ${ }^{1}$, \\ MICHAIL MATALLIOTAKIS ${ }^{2-4}$, IOANNIS MATALLIOTAKIS ${ }^{4}$, DEMETRIOS A. SPANDIDOS ${ }^{5}$, \\ GEORGE N. GOULIELMOS ${ }^{2,6}$ and ELIAS ELIOPOULOS ${ }^{1}$
}

\author{
${ }^{1}$ Laboratory of Genetics, Department of Biotechnology, Agricultural University of Athens, 11855 Athens; \\ ${ }^{2}$ Section of Molecular Pathology and Human Genetics, Department of Internal Medicine, School of Medicine, \\ University of Crete, 71003 Heraklion; ${ }^{3}$ Third Department of Obstetrics and Gynecology, Aristotle University of Thessaloniki, \\ 54124 Thessaloniki; ${ }^{4}$ Department of Obstetrics and Gynecology, 'Venizeleio and Pananio' General Hospital of \\ Heraklion, 71409 Heraklion; ${ }^{5}$ Laboratory of Clinical Virology, School of Medicine, University of Crete, \\ 71003 Heraklion; ${ }^{6}$ Department of Internal Medicine, University Hospital of Heraklion, 71500 Heraklion, Greece
}

Received January 19, 2021; Accepted April 15, 2021

DOI: $10.3892 / \mathrm{ijmm} .2021 .4948$

\begin{abstract}
Demetra Application is a holistic integrated and scalable bioinformatics web-based tool designed to assist medical experts and researchers in the process of diagnosing endometriosis. The application identifies the most prominent gene variants and single nucleotide polymorphisms (SNPs) causing endometriosis using the genomic data provided for the patient by a medical expert. The present study analyzed $>28.000$ endometriosis-related publications using data mining and semantic techniques aimed towards extracting the endometriosis-related genes and SNPs. The extracted knowledge was filtered, evaluated, annotated, classified, and stored in the Demetra Application Database (DAD). Moreover, an updated gene regulatory network with the genes implements in endometriosis was established. This was followed by the design and development of the Demetra Application, in which the generated datasets and results were included. The application was tested and presented herein with whole-exome sequencing data from seven related patients with endometriosis. Endometriosis-related SNPs and variants identified in genome-wide association studies (GWAS), whole-genome (WGS), whole-exome (WES), or targeted sequencing information were classified, annotated and analyzed in a consolidated patient profile with clinical significance information. Probable genes associated with the patient's genomic profile were
\end{abstract}

Correspondence to: Professor Elias Eliopoulos, Laboratory of Genetics, Department of Biotechnology, Agricultural University of Athens, Iera Odos 75, 11855 Athens, Greece

E-mail: eliop@aua.gr

Key words: endometriosis, genome sequencing, exome sequencing, variant analysis, clinical informatics, genomics, bioinformatics, data mining visualized using several graphs, including chromosome ideograms, statistic bars and regulatory networks through data mining studies with relative publications, in an effort to obtain a representative number of the most credible candidate genes and biological pathways associated with endometriosis. An evaluation analysis was performed on seven patients from a three-generation family with endometriosis. All the recognized gene variants that were previously considered to be associated with endometriosis were properly identified in the output profile per patient, and by comparing the results, novel findings emerged. This novel and accessible webserver tool of endometriosis to assist medical experts in the clinical genomics and precision medicine procedure is available at http://geneticslab.aua.gr/.

\section{Introduction}

Endometriosis is a relatively common, enigmatic, benign, estrogen-dependent gynecological illness, characterized by the growth of endometrial tissue and the proliferation of endometrial glands and stroma in ectopic sites, with most common manifestations appearing in the pelvic cavity occurring in sites other than the uterine cavity, most commonly in the pelvic cavity (1). This condition is mainly associated with pelvic pain, dysmenorrhea, dyspareunia and impaired fertility (2). Previous gene association studies, genome-wide association studies (GWAS) and meta-analyses have identified various endometriosis-associated loci, with the list of the novel ones still being enriched $(3,4)$.

Endometriosis markedly affects the health of women, as well as the quality of their life. The gold standard for the diagnosis of endometriosis involves laparoscopy and biopsy, that is, a surgical visual inspection of the pelvic organs, while the development of protocols concerning the treatment of this condition aims for the preservation of patient fertility (5). Advances in modern technologies and bioinformatics have greatly contributed to the generation of large-scale biological 
data, thus leading biomedical sciences to the -omics era. Currently, the search for novel biomarkers for use in endometriosis continues, and the -omics technologies have greatly contributed to this direction. The -omics have revolutionized endometriosis research, and this is proven by the vast number of related publications to date (6). In a recent review, multiple studies based on the high-throughput -omics technologies were presented, in an attempt to gain insight into all considerable advantages that they may confer to proper management of endometriosis (7). The need for non-invasive biomarkers is invaluable and urgent, considering that the average delay between the first symptoms and the laparoscopic diagnosis is estimated at approximately seven years (7). The early diagnosis of endometriosis in combination with proper genetic counseling may facilitate couples to give birth to children at a younger age (of the woman), at an earlier stage of endometriosis, which is characterized by a decreased infertility. Furthermore, the use of non-invasive biomarkers will lead to the elimination of unnecessary laparoscopies (8). According to the current literature, $\sim 5 \%$ of adolescent girls aged between 15-19 experience severe dysmenorrhea not relieved by combined oral contraceptives (COCs) and analgesics, a situation suggestive of endometriosis. Furthermore, other common variable symptoms that may present in young women with endometriosis include dyspareunia in sexually active females, as well as gastrointestinal and urinary tract disturbances (9). Of note, endometriosis is reported to be a differential diagnosis for chronic pelvic pain in adolescent and younger women. Although there are silent (asymptomatic) cases of endometriosis, the majority of symptoms are non-specific and may result in a delay in diagnosis due to the overlapping clinical features with other gynecologic and non-gynecologic conditions. Thus, the early and timely detection of endometriosis with non-invasive procedures may prevent the delay in diagnosis, which can interfere with the quality of life of patients and may result in emotional distress. Moreover, the failure of early recognition and sufficient management may exacerbate the progression of the disease and the development of adhesions that may affect fertility and the risk of the development and maintenance of chronic pain (10).

Advanced techniques in modern genetics and the increasing number of health studies related to genetic and genomic data render precision medicine and consumer genetics a new reality (11). The implementation of a whole-genome (WGS) or whole-exome sequencing (WES) data set as a principal test has provided beneficial information for a more precise diagnosis, aiding and clarifying other conventional tests, while decreasing the number of targeted genetic tests and eventually the time required to perform a full genetic diagnosis (12). The impact of communicating genetic risks is increasingly important for the prevention and treatment of a number of diseases and are rapidly extended to the field of application and practice, as emerging novel genomic pipelines permit more health experts to use information concerning their patients' genetic profiles and gene variants $(11,13)$.

In recent decades, the rapid developments of new technologies in the -omic sciences have produced vast amounts of data. The processing and analysis of such large amounts of data require the understanding of the type of data by inferring structure or generalizations from the data and sophisticated computational analyses towards drawing conclusions (14). The implementation of data mining and semantic techniques in the field of bioinformatics has been widely used for solving such issues, including problem definition, data collection, data annotation, data preprocessing, modeling and validation $(12,15)$. The importance of applying such efficient techniques will grow as researchers continue to generate and integrate large quantities of genomics, proteomics, transcriptomics, lipidomics, metabolomics, secretomics and other-omics biological data. Examples of this type of specialized analyses include GWAS, gene classification based on the literature per disease, the clustering of gene expression data, single nucleotide polymorphism (SNP) classification per disease, regulatory networks of protein-protein interactions, and numerous other applications $(12,16,17)$. The Demetra Application (App) webserver is an example that incorporates the application of bioinformatics and data mining technologies to support the clinical genomic diagnosis process of endometriosis (Fig. 1).

The present study demonstrates the Demetra App toolkit, a webserver capable of facilitating the clinical genomic diagnosis process of endometriosis. The user, by uploading the patient's genetic data to the webserver, either as a FASTA or VCF data file, automatically scans the nucleotide sequence against thousands of relevant recorded SNPs. At the same time, the Demetra App applies different filtering, processing and annotation techniques, towards identifying and visualizing the most probable dominant and relevant variants related to endometriosis. The Demetra App toolkit identifies and classifies all the candidate SNPs using an up-to-date curated database with SNPs and other clinical information, and provides those gene variants and SNPs with probably functional pathogenic effects in endometriosis, guided by explanatory information and direct links to several online databases such as the dbSNP and LitVar databases $(18,19)$. Additionally, the Demetra App extracts and exports other important information related to the identified variants in the patient's profile, including chromosome ideograms, statistics bars, a regulatory gene networks, and several relevant publications from the PubMed database.

\section{Data and methods}

Demetra App Database (DAD) of SNPs and variants for endometriosis. The DAD aimed to develop a resource with all genes, SNPs and variants associated with endometriosis reported in the online databases and the literature. The PubMed database depository was initially mined in order to detect and extract entries related to 'endometriosis'. The query was limited to human studies only. The articles retrieved were curated using data mining techniques aimed towards identifying those containing gene names by using a dictionary from the gene database of the National Center for Biotechnology Information (NCBI) (20). A search query was built using regular expressions by combining each gene or variant with their synonyms and the keyword 'endometriosis' (21). The extracted genes, SNPs and variants referred in the article dataset were stored in DAD. Furthermore, each relevant PubMed reference abstract was mined for the provision of additional information, such as MeSH/MEDLINE terms, polymorphisms/mutations described and other genes 


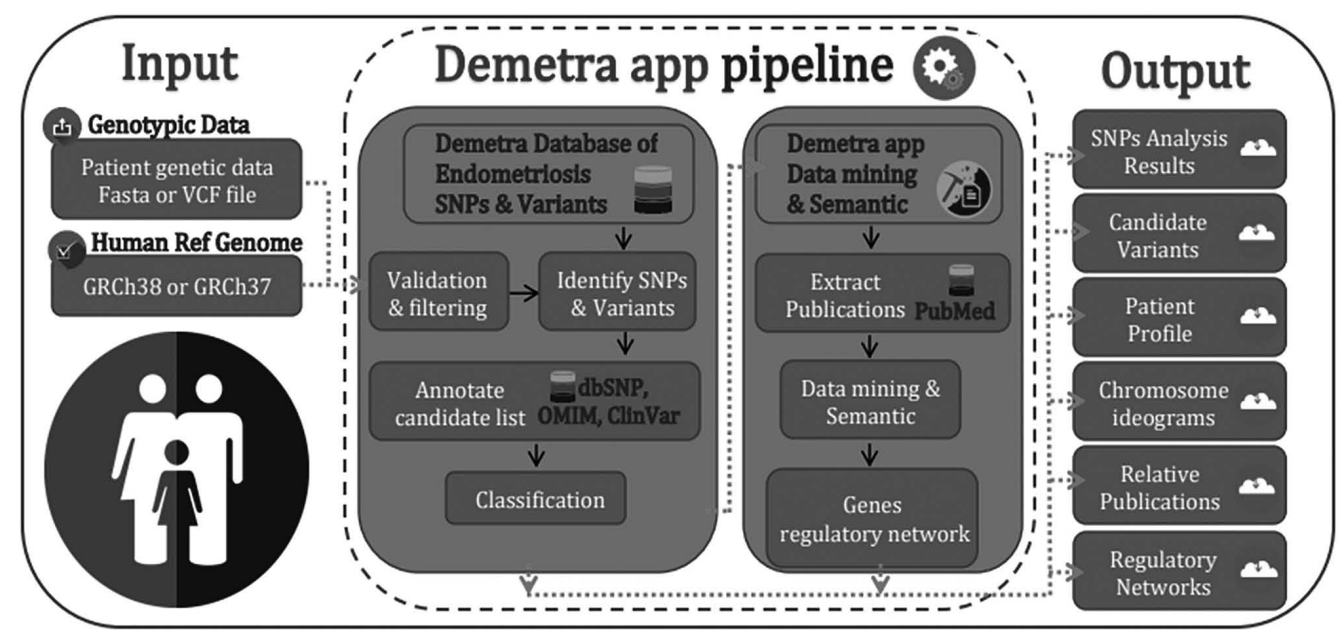

Figure 1. Demetra Application webserver pipeline. Left to right: Input parameters (FASTA or VCL file and a selected reference genome), Demetra Application pipeline, Output files (SNP analysis results, candidate variants, patient profile and statistic charts, chromosome ideograms, relative publications with candidate variants and regulatory network). SNP, single nucleotide polymorphism.

in the reference studied for their role in endometriosis (21). Additional information was extracted and added to the DAD from several available online databases, including the Online Mendelian Inheritance in Man (OMIM) database (22) and Endometriosis Knowledgebase (3). All the extracted SNPs and variants associated with endometriosis and contained in the DAD were annotated using key terms and external searches in the dbSNP, ClinVar and LitVar databases of the NCBI $(18,19,24)$, and representative FASTA files were generated using the human reference genome, GRCh38, and the human mitochondrial complete genome (NCBI: NC_012920.1). Preset windows of 201 bases (100 before and 100 after the change/deletion or insertion of the polymorphism) were applied to the corresponding genetic locus of each identified SNPs and representative FASTA files were generated. Finally, the information contained in the DAD was classified according the scoring function described below and the final outcome was manually evaluated by medical experts in endometriosis using the annotated information, results and the sources of origin as follows:

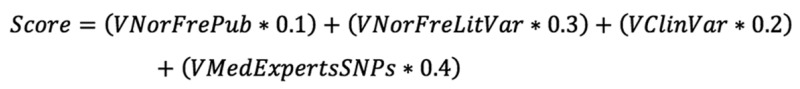

Scoring Function $=$ Strong - associated SNPs Class: Score $>0.4$,

$$
\begin{aligned}
& \text { High - associated SNPs Class: } 0.4>\text { Score } \geq 0.2 \\
& \text { Associated SNPs Class: } 0.2>\text { Score }
\end{aligned}
$$

where VNorFrePub represents the normalized frequency of the identified SNPs from the PubMed dataset (Max = 1/Min =0); VNorFreLitVar represents the normalized frequency of the identified SNPs based on the LitVar database output and endometriosis connections (Scalar value, $\operatorname{Max}=1 / \mathrm{Min}=0$ ); VClinVar represents the Boolean Parameter (1 indicates that the SNP was identified in the ClinVar database and has a connection with endometriosis; 0 indicates that there is no profile in the ClinVar database, or no connection to endometriosis); and VMedExperts represents the Boolean Parameter (1 indicates that the given SNP has been characterized as beiong associated with the endometriosis by the medical experts team; and 0 indicates equal to no connection.
VCF or FASTA file validation and filtering. The uploaded file is validated for compliance with the standardized formats including, FASTA format or VCF format four, correspondingly (5). The FASTA headers should contain the genetic data labels and key terms and the genetic information sequence in a string of nucleotides $>250$ characters. FASTA entries must begin with the symbol ' $>$ ', and a tab separated at the end, have each the suitable data type, and have no duplicated header string names. Respectively, the VCF header should include the format information and the defined column names as they specified by the Global Alliance for Genomics and Health (https://www.ga4gh.org/) (5). VCF file columns must be separated with tabs, have no duplicated entries and each entry must contain only the proper data type without gaps. In this initial version, the file size that can be uploaded to the Demetra webserver must be $\leq 300 \mathrm{MB}$. In the next step of analysis in the Demetra webserver pipeline, only SNPs and gene variants that have passed the quality and filtering controls will be considered as an input structured database.

Identification of SNPs and variants. The Demetra App webserver has two different SNP and variant identification processes depending on the type of the uploaded file (FASTA of VCF file). For each pipeline of the two main processes, the webserver uses the DAD of SNPs and variants associated with endometriosis to analyze and correlate the input curated dataset. In the case of a FASTA file, the application implements the process of the local alignments with the DAD. Input entries identified with $100 \%$ identity in a range of a window of 200 bases within a given nucleotide sequence from DAD are reported and marked to the system as a candidate mutation case endometriosis. In the second case of the VCF file, all the endometriosis-related SNPs and variants are identified based on the DAD's directory with the reported positions of SNPs and variants on each chromosome. Finally, all the identified cases in each case of the analysis are collected in a separated list with all the annotated information from the DAD.

Variant classification and interface representation. The Demetra App classification procedure identifies the most 


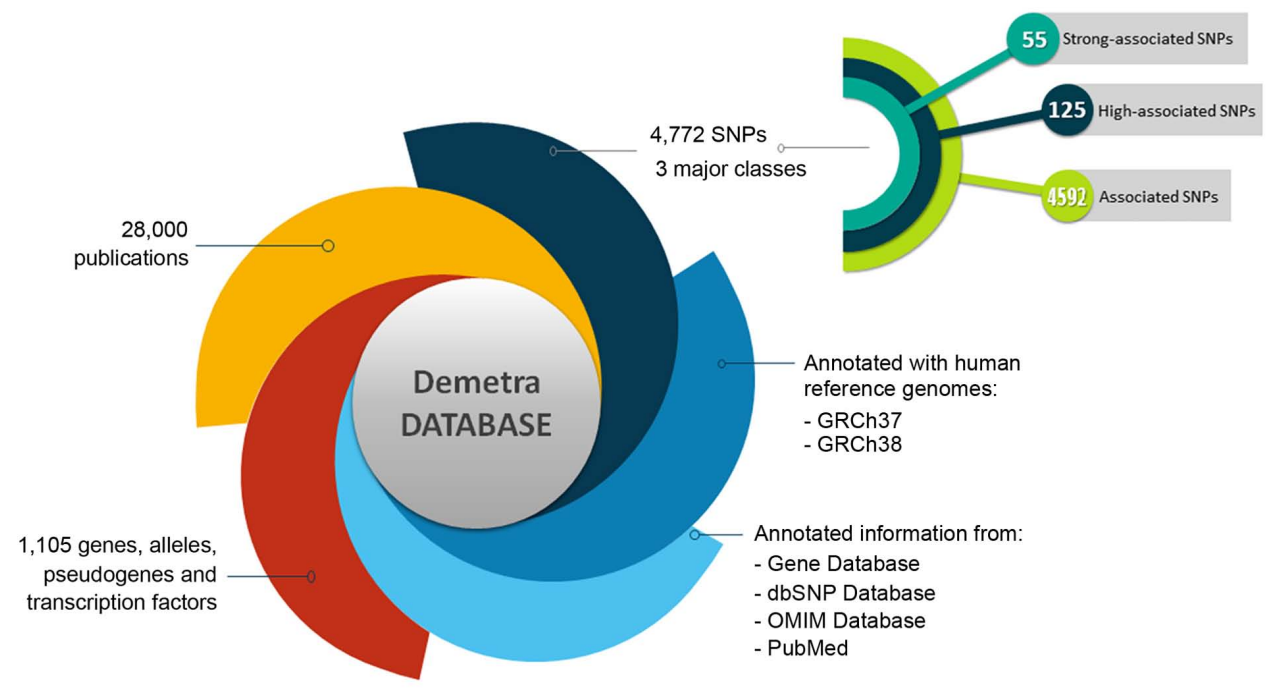

Figure 2. Demetra Application endometriosis database. SNP, single nucleotide polymorphism; OMIM, Online Mendelian Inheritance in Man.

candidate and dominant deleterious SNPs and gene variants in the list of exonic and non-coding polymorphisms. The graphic representation interface enables the user to see the patient endometriosis profile, which is presented through the three major classes of polymorphisms according to the application scoring function, namely 'Strong-associated SNPs', 'High-associated SNPs', and 'Associated SNPs'. All the identified SNPs are classified in these three major classes based on the annotated information contained in the DAD. An additional list of all identified variants with necessary information, such as 'snp_ name', 'chromosome', 'position', 'reference genome', 'change', 'gene_name', 'variant_type', 'disease', 'litvar' and 'class' is also provided to the user. Moreover, for each identified variant, the application provides an external link to the dbSNP and LitVar databases for reference to additional information.

A more specialized representation with chart bars and chromosome ideograms is presented based on the patient's identified polymorphism profile. This enables the user to better understand the general genetic profile for the patient, as well as to draw beneficial conclusions about the association of each chromosome in endometriosis development. With this more specialized analysis, conclusions can be drawn on how genes may be involved in endometriosis, not only as separate entities, but as part of specific chromosomal regions or as a cluster in a network or in a combination of both.

Data mining and semantics. The MEDLINE and PubMed databases were searched for English-language publications that contain the key term 'endometriosis', with no date restrictions (21). The Matlab Bioinformatics toolbox functions for data mining and semantics were used to extract gene names from the abstracts of the selected publications using a dictionary of the gene, allele and pseudogene names for Homo sapiens (17,26). Furthermore, using the same techniques, all the polymorphisms reported by at least two studies from the dataset were extracted. A second-level analysis was performed in order to estimate the internal links between genes through selected publications. Internal links were created when genes, alleles, pseudogenes, or transcription factors were mentioned in the same publication. Finally, all the mining knowledge was processed through semantic algorithms contained in the Matlab 'Data Analysis for Computational Biology', towards estimating correlations among genes and generating the regulator network in a graph representation for endometriosis (26-28).

Demetra App web server security and availability. The Demetra App web tool was used on a Secure XAMPP HTTP Apache webserver hosted on the computing facility of the School of Applied Biology and Biotechnology at the Agricultural University of Athens (AUA). All DADs and third-party software packages used are locally installed, and thus there are no additional information transferred to other webservers. The user genomic data uploaded in the webserver are used for the Demetra App pipeline only, while the results are stored privately and securely for a period of three months and subsequently deleted afterward. The pipeline for identifying the most probable SNPs and gene variants causing endometriosis described above is executed in the webserver named, Demetra Application web tool, using Windows, Apache, XAMPP, PHP, HTML, JavaScript, R and parallel computing architecture, and is openly available online at http://geneticslab.aua.gr/.

\section{Results}

Demetra App. The Demetra App endometriosis database is an integrated resource for genes, alleles, pseudogenes, transcription factors and SNPs associated with endometriosis. The information and the several fields of knowledge contained in the DAD were evaluated and classified based on the novel pipeline and the specific scoring function were descripted in the present study. The DAD currently holds information on 1,105 genes, alleles, pseudogenes and transcription factors, 4,772 SNPs and 28,000 related publications (Fig. 2). Moreover, 68 SNPs were detected in the coding region sites of genes (Fig. 3).

All the SNPs associated with endometriosis were manually curated and classified into three major classes, including 'strong-associated SNPs' with 55 members, 'high associ- 
A

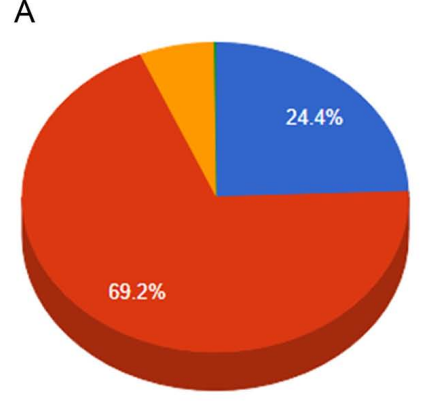

C

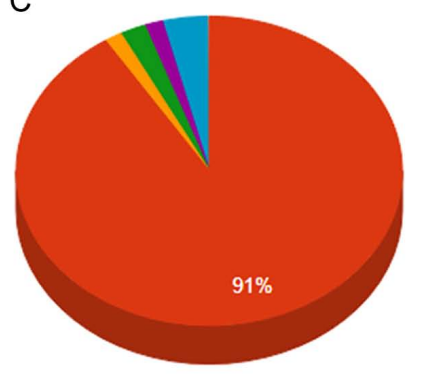

N/A

$\times 1$

$\times 2$

$\times 3$

Intron variant

Missense,

synonymous \& splice acceptor variant

Non coding transcript variant

3 or 5 Prime UTR variant

$2 \mathrm{~KB}$ upstream \& $500 \mathrm{~B}$ downstream variant

Other
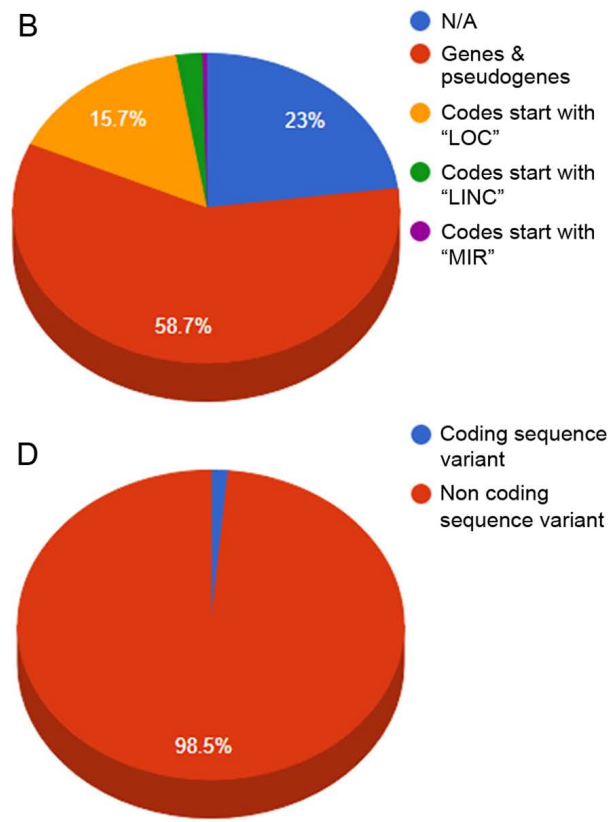

Coding sequence variant

- Non coding sequence variant

Figure 3. Database analysis results. (A) ' $\mathrm{x} 1$ ', ' $\mathrm{x} 2$ ' and ' $\mathrm{x} 3$ ' correspond to the number of the affected regions per SNP. (B) The five identified categories within the Demetra Database. (C) The identified types of SNPs within the Demetra Database. (D) The two major categories of the genomic regions identified categories within the Demetra Database. SNP, single nucleotide polymorphism.

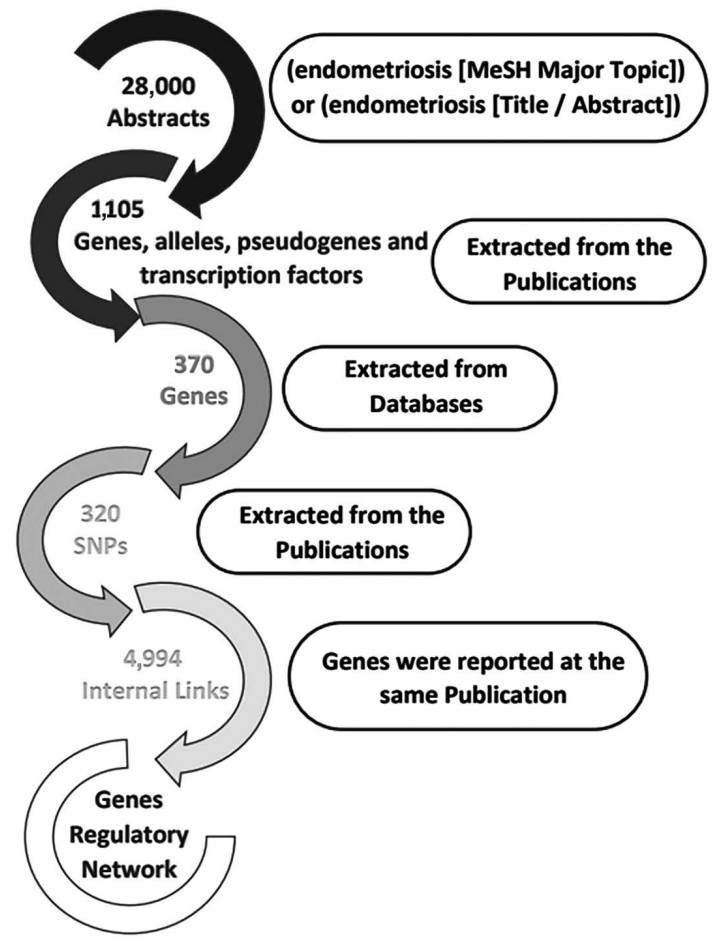

Figure 4. Selection of genes, alleles, pseudogenes and transcription factors for data mining and semantic analysis.

ated SNPs' with 125 members and 'associated SNPs' with 4,592 members (Fig. 2). Moreover, each polymorphism is described by a nucleotide sequence of $\sim 200$ bases using the Homo sapiens reference genome, GRCh38. The database also includes information from the Gene Database, dbSNP Database, LitVar Database, ClinVar Database, OMIM Database and PubMed Database. The information within the database is structured in several fields, and the knowledge is organized in a specific manner in order to serve the webserver application immediately and efficiently (Fig. 3).

Data mining and semantic analysis for endometriosis. A systematic data mining and semantic analysis of the most regularly reported genes and polymorphisms was performed in order to identify those that may play a critical role in endometriosis and may thus be of value in clinical genomics. For the purpose of the present study, 28,000 publications were analyzed, which contained the term 'endometriosis' in the title or abstract of the MEDLINE file. In the first level of the analysis, 1,105 gene, allele, pseudogene and transcription factor names or synonyms were identified, and 430 key terms were describing endometriosis, which was present in $>10$ publications within the dataset (Fig. 4). The 30 most frequently identified key terms describing endometriosis are presented in Table I. Moreover, within the dataset, 320 different SNPs and 370 relative genes with endometriosis were reported and imported from online databases. Therefore, the analysis allowed the identification of polymorphisms that could potentially be included in the DAD, alongside the other SNPs that could definitely predispose to endometriosis. In the second level of analysis, 4,994 internal links among genes, alleles, pseudogenes and transcription factors were estimated through publications, and the regulatory network was calculated in a graph representation (Fig. 3). The major goal of this step of the analysis was to provide an exhaustive regulatory network in genes where are directly related to endometriosis (Fig. S1).

The extracted knowledge from the data mining and semantic analysis for endometriosis is included in the Demetra App in a seamless way, where for each patient profile, the pre-analyzed information is used towards drawing the corresponding gene regulatory network based on the identified genes from the SNPs results. The Demetra App webserver contains all the pre-analyzed data in an effort to calculate and draw the 
Table I. List of the 30 most frequently shown key terms describing endometriosis within the dataset.

\begin{tabular}{rlll}
\hline A/A & \multicolumn{1}{c}{ Key term } & A/A & \multicolumn{1}{c}{ Key term } \\
\hline 1 & Laparoscopy & 16 & Genitalia \\
2 & Infertility & 17 & Hysterectomy \\
3 & Endometrium & 18 & Ovarian cancer \\
4 & Endometrioma & 19 & Ovary \\
5 & Family planning & 20 & Fertility \\
6 & Pelvic pain & 21 & Reproduction \\
7 & Pregnancy & 22 & Ovarian reserve \\
8 & Contraception & 23 & Deep endometriosis \\
9 & Dysmenorrhea & 24 & Endometriosis/complications \\
10 & Uterus & 25 & Uterine neoplasms \\
11 & Adenomyosis & 26 & Apoptosis \\
12 & Deep infiltrating endometriosis & 27 & Hormones \\
13 & Research methodology & 28 & Endocrine system \\
14 & Urogenital system & 29 & Endometrial effects \\
15 & Inflammation & 30 & Angiogenesis \\
\hline
\end{tabular}

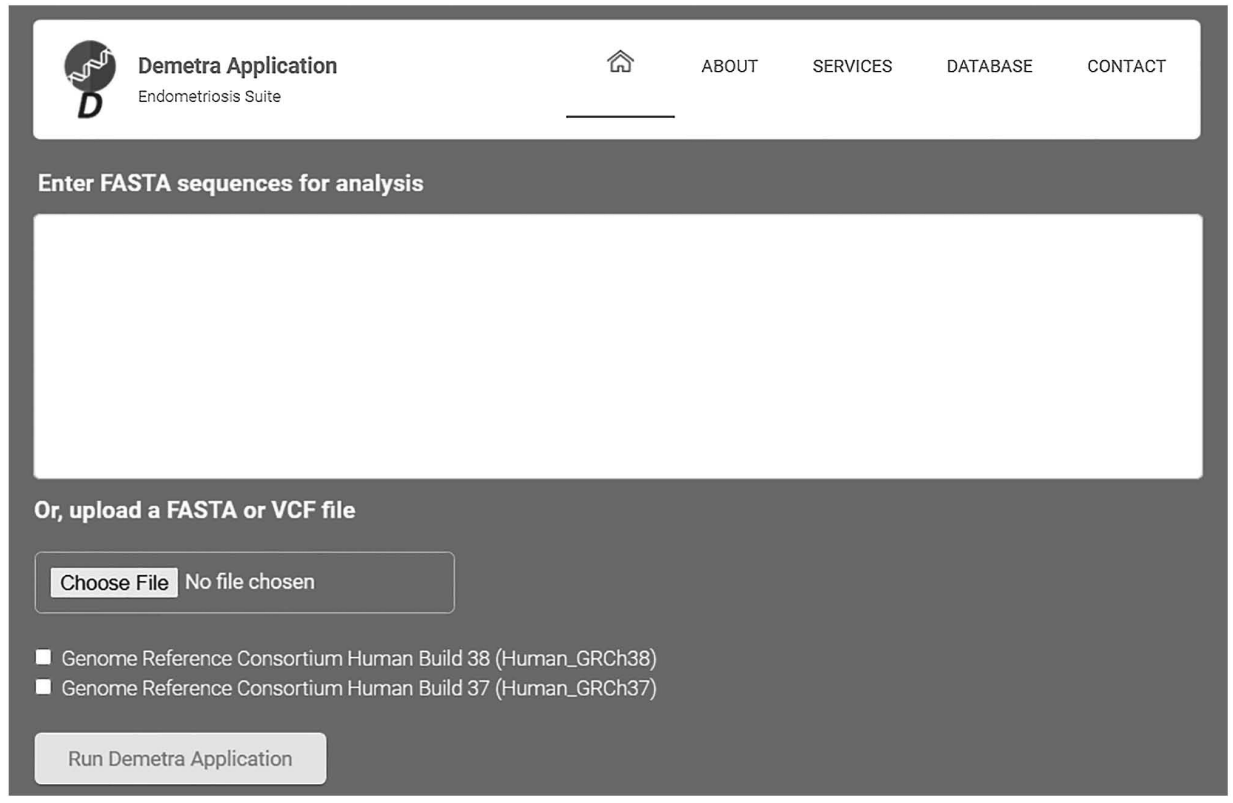

Figure 5. Demetra Application user interface.

regulatory gene network of each patient. The application generates a personalized regulatory network graph based on patient profile using all the identified SNPs related to genes, alleles, pseudogenes and transcription factors from the previous steps of the described pipeline. Thus, in addition to the detected polymorphisms, the Demetra App is capable of returning a list of the genes directly involved in several biological processes with the reference identified genes. Furthermore, beyond the generated graph, all the internal links are provided in a list along with genes and relative publications.

Demetra App webserver. The Demetra App webserver assists the health expert in confirming an endometriosis diagnosis for a patient using genetic information. This effective and time-consuming otherwise pipeline has been designed by geneticists able to benefit from bioinformatics support and by medical experts in endometriosis aiming to evaluate and classify all the determined variants and genes related to endometriosis. Due to the large amount of data required to be analyzed and the computational complexity of this pipeline, advanced bioinformatics techniques and parallel programming have been applied. It is estimated that using a parallel programming webserver requires much less time (10-fold) to analyze and extract the final results. Based on various tests executed on the performance of this application, it was estimated that this webserver has the ability to analyze a VCF file of 37,000 variants and create a personalized patient profile in $<20 \mathrm{~min}$. The Demetra App has been designed to reduce 
Demetra Application

Endometriosis Suite

Demetra App/ Endometriosis suite / results for "Greece_4_lonXpress.vcf"

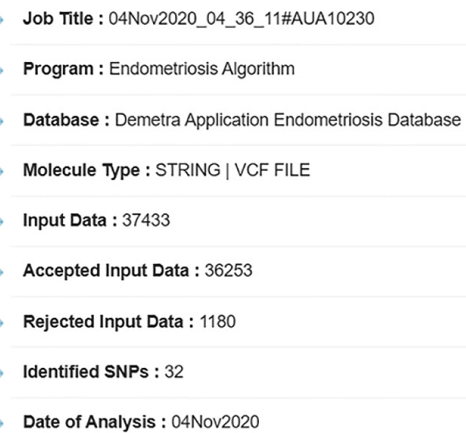

\section{SNPs Analysis Results for Endometriosis}

Strong-associated SNPS

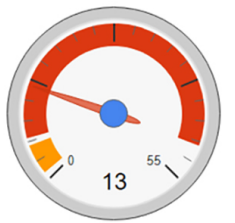

Show $10 \sim$ entries

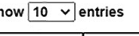

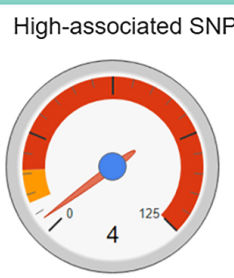

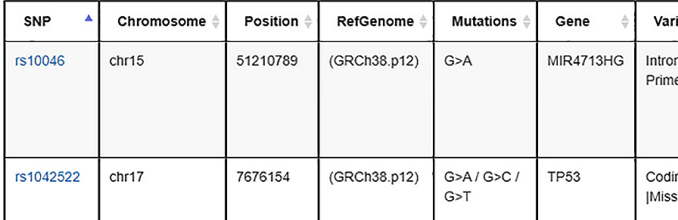

\begin{tabular}{|c|c|c|c|c|c|c|}
\hline rs1042522 & chr17 & 7676154 & (GRCh38.p12) & $\begin{array}{l}G>A / G>C / \\
G>T\end{array}$ & TP53 & $\begin{array}{l}\text { Coding Sequence Variant } \\
\text { |Missense Variant }\end{array}$ \\
\hline is 10455097 & chr6 & 73783709 & (GRCh38.p12) & $A>C$ & CD109 & Coding Sequence Variant \\
\hline
\end{tabular}

\begin{tabular}{|l|l|l|l|l|l|l|}
\hline rs1056836 & chr2 & 38071060 & (GRCh38.p12) & G>C & CYP181 & $\begin{array}{l}\text { Coding Sequence variant } \\
\text { IMissense Variant }\end{array}$ \\
\hline
\end{tabular}

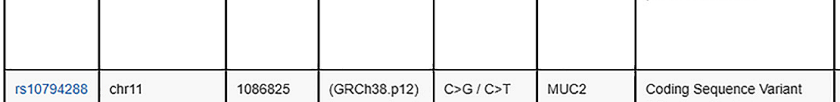

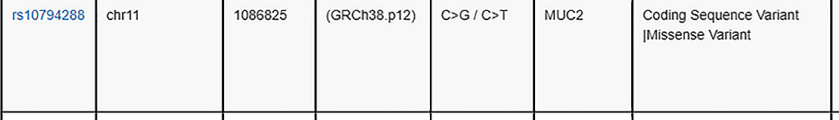

\begin{tabular}{|c|c|c|c|c|c|c|c|c|c|}
\hline rs10929757 & chr2 & 11562535 & (GRCh38.p12) & $A>C$ & GREB1 & $\begin{array}{l}\text { Coding Sequence Variant } \\
\text { |Missense Variant }\end{array}$ & $\begin{array}{l}\text { Leiomyoma }=2 \\
\text { Endometriosis }=1 \\
\text { AMRSyndrome }=1 \\
\text { Atrialfibrillation }=1\end{array}$ & rs 10929757 & c \\
\hline rs1135352 & chr1 & 214369476 & (GRCn38.p12) & $T>A / T>C$ & PTPN14 & $\begin{array}{l}\text { Coding Sequence Variant } \\
\text { |Missense Variant }\end{array}$ & & & c \\
\hline rs11549465 & chr14 & 61740839 & (GRCh38.p12) & $C>T$ & HIF1A & $\begin{array}{l}\text { Coding Sequence Variant } \\
\text { |Missense Variant||HIF1A- } \\
\text { AS3||ntron Variant }\end{array}$ & $\begin{array}{l}\text { Neoplasms }=355 \\
\text { BreastNeoplasms }=116 \\
\text { ProstaticNeoplasms }=98 \\
\text { Gliomal }=58 \\
\text { MultipleMyeloma }=54\end{array}$ & rs11549465 & A \\
\hline rs12470971 & chr2 & 11585115 & (GRCh38.p12) & $G>A / G>C$ & GREB1 & Intron Variant & $\begin{array}{l}\text { PolycysticovarySyndrome }=1 \\
\text { Endometriosis }=1 \\
\text { OvarianNeoplasms }=1\end{array}$ & rs 12470971 & c \\
\hline rs 1250259 & $\mathrm{chr} 2$ & 215435759 & (GRCh38.p12) & $T>A$ & $\mathrm{FN} 1$ & $\begin{array}{l}\text { Missense } \\
\text { Variant||LOC105373868|2KB } \\
\text { Upstream Variant }\end{array}$ & & & c \\
\hline
\end{tabular}

Showing 1 to 10 of 32 entries

Associated SNPs

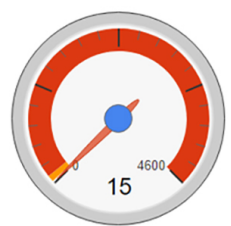

Search:

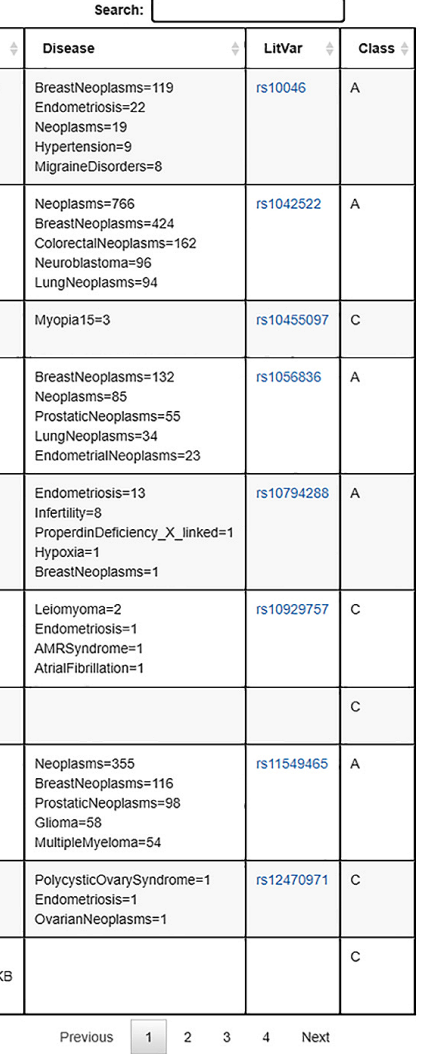

Figure 6. Demetra Application output interface, part 1.

complexity and minimize probable errors, allowing health experts to inset only a patient's genomic data from FASTA or VCF file towards estimating a clear and concise output HTML file with the patient profile (Fig. 5).
The Demetra App is a state-of-the-art webserver, designed for health experts in the scientific field of medicine and clinical genomics who may not have advanced skills in computers to filter, classify and annotate SNPs variants 


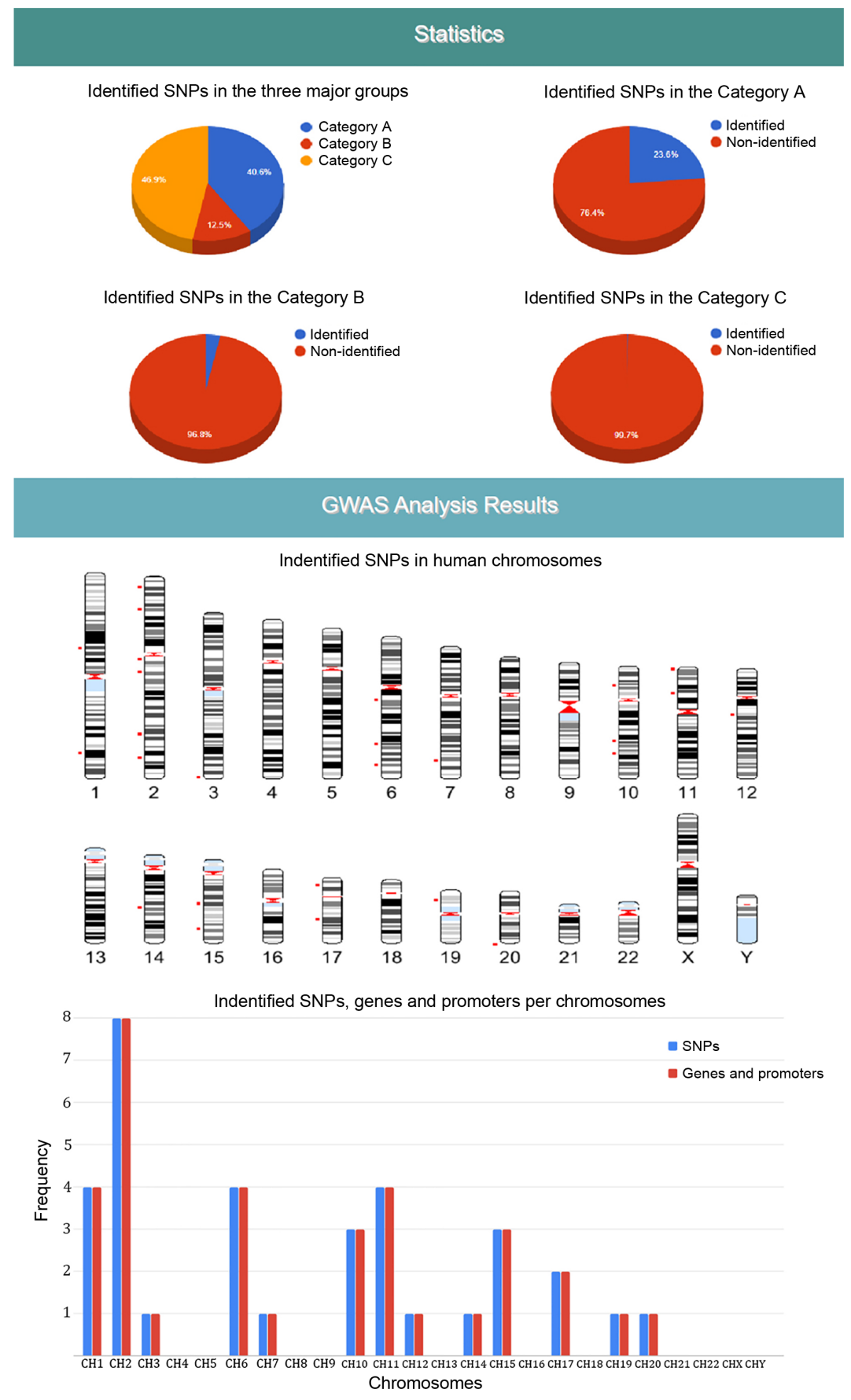

Figure 7. Demetra Application output interface, part 2.

recognized in sequencing studies, to be allowed to choose and summarize the SNPs and gene variants that are associated with endometriosis. The Demetra App output is an HTML file that describes the patient profile through six major areas of results, including 'Server output details', 'SNPs Analysis Results for Endometriosis', 'Statistic Charts', 'GWAS Analysis Results', 'Semantic and Data mining of identified Genes' and 'Downloads' (Figs. 6-8). In the first results section, a summary of the analyzed information is presented including, the type of the data file analyzed, the number of the identified SNPs, and the date the analysis was performed (Fig. 6). In the second section, the results of the SNP classification are shown in three separated charts and a list of all identified SNPs with extra information for each SNP as extracted from the DAD (Fig. 6).
The third results section is concerned with various statistics charts regarding identified SNPs and the overall SNPs contained in the DAD (Fig. 7). The fourth section provides GWAS analysis results in a graphical representation of the chromosome ideogram, where all the identified SNPs in each genetic locus per chromosome have been marked. Moreover, a statistical chart indicating the identified SNPs per chromosome (Fig. 7) is shown. In the sixth section, the results from the data mining and semantic analysis are presented (Fig. 8). A list of all identified genes is provided with all the information mined from the relative publications towards calculating and drawing the regulatory network in a graph representation. The user can filter the list in several ways and has the option to retrieve the relevant publications that describe each internal 


\section{Semantic and Data mining of identified genes}

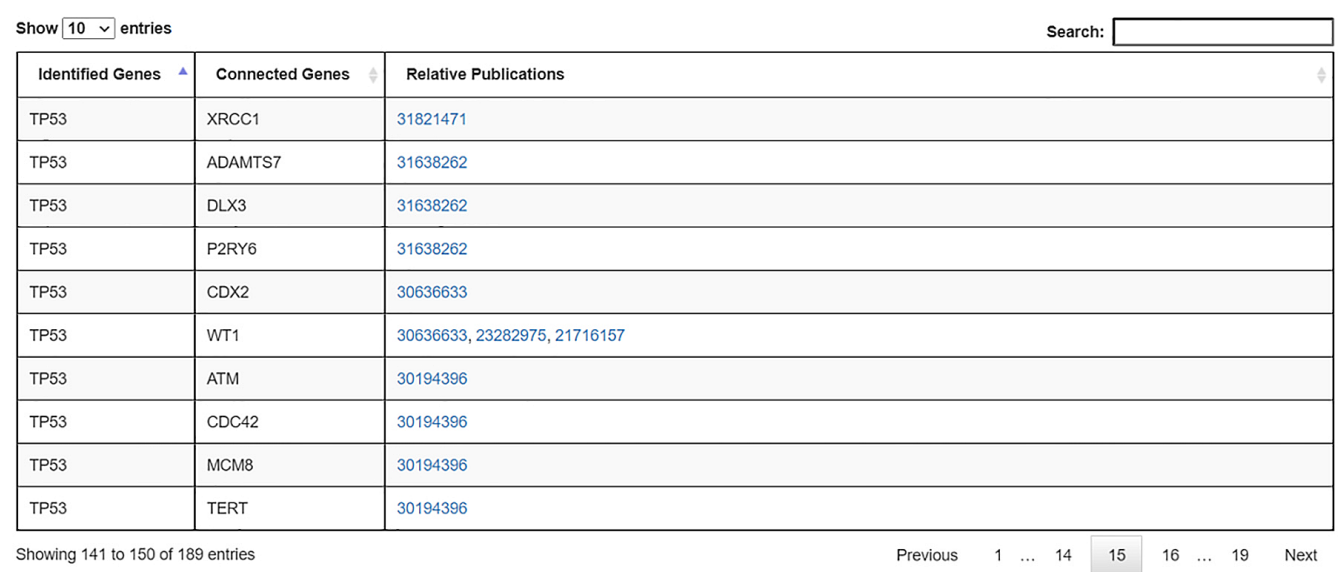

Extracted genes from data mining analysis in graph

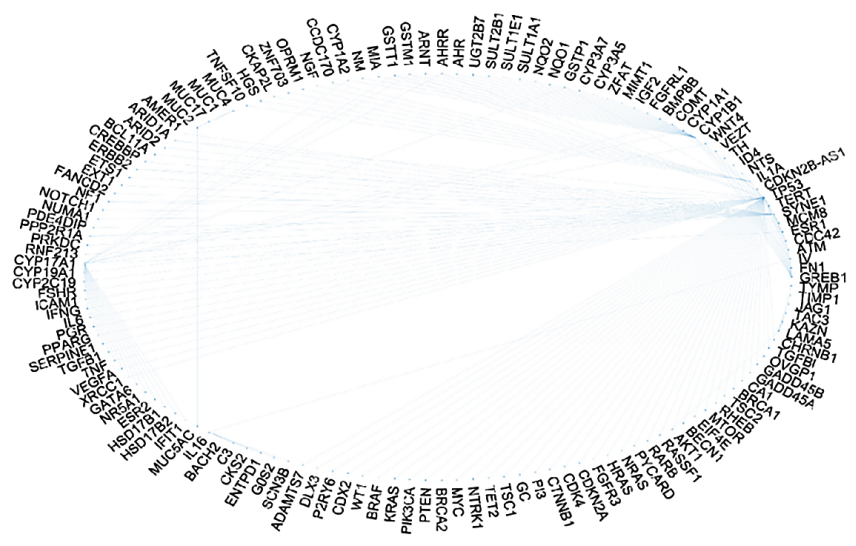

Downloads
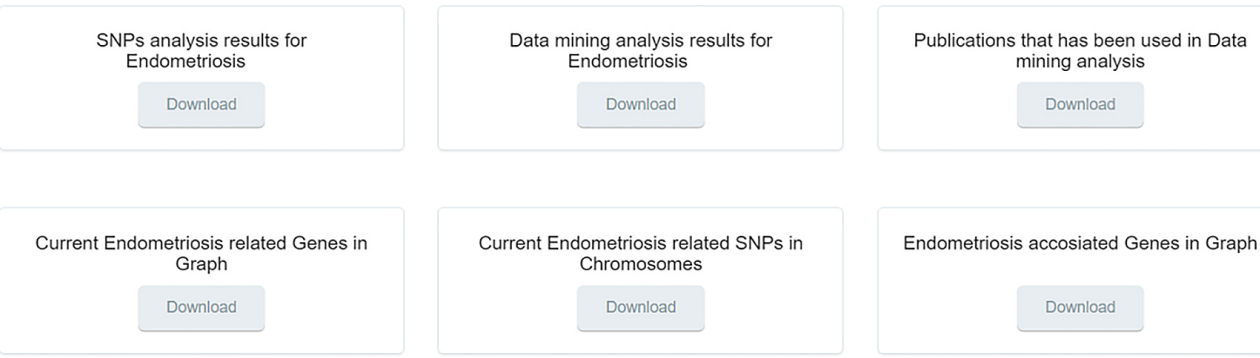

Endometriosis accosiated Genes in Graph

Download

Figure 8. Demetra Application output interface, part 3.

link within the network. Moreover, the beneficial knowledge of all connected genes with the identified genes is provided to the users. In the last results section, the user has the choice to download and save all the generated results from the DAD webserver (Fig. 8).

Demetra App validation. Demetra App webserver validation was performed by a retrospective study performed by Albertsen et al (29) on seven patients from a three-generation family with endometriosis from the 'Venizeleio and Pananio' General Hospital of Heraklion, Greece. The WES data of the seven patients presented in the study by Albertsen et al (29) in detail, were reanalyzed using the Demetra App webserver. A list with all known genes that were previously reported as 'endometriosis-associated' was properly identified in the final output HTML profile per patient, and by cross-comparison of the results, new findings have emerged. The SNPs analysis performed identified the common pathogenic variants that 
Table II. Major SNP cases identified in the seven patients with endometriosis.

\begin{tabular}{|c|c|c|c|c|c|c|c|}
\hline SNP & Chromosome & Change & Gene & Type & Class & Patients & Frequency \\
\hline rs 1056836 & $\operatorname{chr} 2$ & $\mathrm{G}>\mathrm{C}$ & CYP1B1 & Coding sequence variant & A & $01|02| 03|04| 05|06| 07$ & 7 \\
\hline rs13394619 & $\operatorname{chr} 2$ & $\mathrm{G}>\mathrm{A}$ & GREB1 & Splice acceptor variant & A & $01|02| 03|04| 05|06| 07$ & 7 \\
\hline rs 2258447 & $\operatorname{chr} 3$ & $\mathrm{~T}>\mathrm{A} / \mathrm{T}>\mathrm{C}$ & MUC4 & Coding sequence variant & A & $01|02| 03|04| 05|06| 07$ & 7 \\
\hline rs700518 & $\operatorname{chr} 15$ & $\mathrm{~T}>\mathrm{C}$ & CYP19A1 & Coding sequence variant & A & $01|02| 03|04| 05|06| 07$ & 7 \\
\hline rs 1042522 & $\operatorname{chr} 17$ & $\mathrm{G}>\mathrm{C} / \mathrm{G}>\mathrm{T}$ & TP53 & Coding sequence variant & A & $01|02| 03|04| 05|06| 07$ & 7 \\
\hline rs 2427284 & $\operatorname{chr} 20$ & $\mathrm{~A}>\mathrm{G} / \mathrm{A}>\mathrm{T}$ & LAMA5 & Coding sequence variant & A & $01|02| 03|04| 05|06| 07$ & 7 \\
\hline rs 10794288 & $\operatorname{chr} 11$ & $\mathrm{C}>\mathrm{G} / \mathrm{C}>\mathrm{T}$ & MUC2 & Coding sequence variant & A & $01|02| 03|04| 06 \mid 07$ & 6 \\
\hline rs743572 & chr10 & $\mathrm{A}>\mathrm{G} / \mathrm{A}>\mathrm{T}$ & CYP17A1 & 5 Prime UTR variant & A & $01|02| 03|04| 07$ & 5 \\
\hline rs 10046 & $\operatorname{chr} 15$ & $\mathrm{G}>\mathrm{A}$ & MIR4713HG & Intron variant & A & $01|02| 03|04| 06$ & 5 \\
\hline rs2304402 & $\operatorname{chr} 2$ & $\mathrm{G}>\mathrm{A}$ & GREB1 & Coding sequence variant & A & $01|02| 03|04| 07$ & 5 \\
\hline rs 11549465 & $\operatorname{chr} 14$ & $\mathrm{C}>\mathrm{T}$ & IF1A & Coding sequence variant & A & $01|02| 03 \mid 04$ & 4 \\
\hline rs1799930 & chr8 & $\mathrm{G}>\mathrm{A}$ & NAT2 & Coding sequence variant & A & 01102103 & 3 \\
\hline rs4072111 & $\operatorname{chr} 15$ & $\mathrm{C}>\mathrm{T}$ & IL16 & Coding sequence variant & A & 04105 & 2 \\
\hline rs5498 & chr19 & $\mathrm{A}>\mathrm{G}$ & ICAM1 & Coding sequence variant & $\mathrm{B}$ & $01|02| 03104|05| 06$ & 6 \\
\hline rs 3783550 & $\operatorname{chr} 2$ & $\mathrm{G}>\mathrm{T}$ & IL1A & Intron variant & $\mathrm{B}$ & $01|02| 03|04| 06$ & 5 \\
\hline rs7103978 & $\operatorname{chr} 11$ & $\mathrm{~A}>\mathrm{G} / \mathrm{A}>\mathrm{T}$ & MUC2 & Coding sequence variant & $\mathrm{B}$ & 01103104107 & 4 \\
\hline rs113759408 & chr8 & $\mathrm{G}>\mathrm{A}$ & CYP11B1 & Intron variant & $\mathrm{B}$ & 02103 & 2 \\
\hline rs 280523 & chr19 & $\mathrm{G}>\mathrm{A} / \mathrm{G}>\mathrm{C}$ & TYK2 & Coding sequence variant & $\mathrm{B}$ & 01107 & 2 \\
\hline rs 1801133 & chr1 & $\mathrm{G}>\mathrm{A}$ & MTHFR & Coding sequence variant & $\mathrm{B}$ & 03107 & 2 \\
\hline rs 1802669 & $\operatorname{chr} 10$ & $\mathrm{G}>\mathrm{A} / \mathrm{G}>\mathrm{T}$ & MLLT10 & Coding sequence variant & $\mathrm{B}$ & 01104 & 2 \\
\hline rs605059 & $\operatorname{chr} 17$ & $\mathrm{G}>\mathrm{A} / \mathrm{G}>\mathrm{C} / \mathrm{G}>\mathrm{T}$ & HSD17B1 & Coding sequence variant & $\mathrm{B}$ & 01 & 1 \\
\hline rs500760 & $\operatorname{chr} 11$ & $\mathrm{~T}>\mathrm{C}$ & PGR & Coding sequence variant & $\mathrm{B}$ & 06 & 1 \\
\hline rs 2304256 & chr19 & $\mathrm{C}>\mathrm{A}$ & TYK2 & Coding sequence variant & $\mathrm{B}$ & 06 & 1 \\
\hline rs 12720270 & chr19 & $\mathrm{G}>\mathrm{A}$ & TYK2 & Intron variant & $\mathrm{B}$ & 06 & 1 \\
\hline rs 1135352 & $\operatorname{chr} 1$ & $\mathrm{~T}>\mathrm{C}$ & PTPN14 & Coding sequence variant & $\mathrm{C}$ & $01|02| 03|04| 05|06| 07$ & 7 \\
\hline rs3013451 & $\operatorname{chr} 1$ & $\mathrm{G}>\mathrm{A}$ & PTPN14 & Intron variant & $\mathrm{C}$ & $01|02| 03|04| 05|06| 07$ & 7 \\
\hline rs7550799 & chr1 & $\mathrm{T}>\mathrm{A} / \mathrm{T}>\mathrm{C}$ & PTPN14 & Coding sequence variant & $\mathrm{C}$ & $01|02| 03|04| 05|06| 07$ & 7 \\
\hline rs 2241820 & $\operatorname{chr} 12$ & $\mathrm{C}>\mathrm{A} / \mathrm{C}>\mathrm{T}$ & HOXC9 & Coding sequence variant & $\mathrm{C}$ & $01|02| 03|04| 05|06| 07$ & 7 \\
\hline rs10929757 & $\operatorname{chr} 2$ & $\mathrm{~A}>\mathrm{C}$ & GREB1 & Coding sequence variant & $\mathrm{C}$ & $01|02| 03|04| 05 \mid 06$ & 6 \\
\hline rs12470971 & $\operatorname{chr} 2$ & $\mathrm{G}>\mathrm{A}$ & GREB1 & Intron variant & $\mathrm{C}$ & $01|02| 03|04| 05 \mid 06$ & 6 \\
\hline rs1250259 & $\operatorname{chr} 2$ & $\mathrm{~T}>\mathrm{A}$ & FN1 & Missense variant & $\mathrm{C}$ & $01|02| 03|04| 05 \mid 06$ & 6 \\
\hline rs 2278868 & $\operatorname{chr} 17$ & $\mathrm{C}>\mathrm{T}$ & SKAP1 & Coding sequence variant & $\mathrm{C}$ & 01102104105106107 & 6 \\
\hline rs7586970 & $\operatorname{chr} 2$ & $\mathrm{~T}>\mathrm{C} / \mathrm{T}>\mathrm{G}$ & FPI & Coding sequence variant & $\mathrm{C}$ & $01|02| 03|04| 07$ & 5 \\
\hline rs6973420 & $\operatorname{chr} 7$ & $\mathrm{~A}>\mathrm{G}$ & CALD1 & Coding sequence variant & $\mathrm{C}$ & $01|02| 03|04| 07$ & 5 \\
\hline rs2918308 & chr19 & $\mathrm{A}>\mathrm{C}$ & NFILZ & 3 Prime UTR variant & $\mathrm{C}$ & $02103104 \mid 05$ & 4 \\
\hline rs6169 & $\operatorname{chr} 11$ & $\mathrm{C}>\mathrm{T}$ & FSHB & Coding sequence variant & $\mathrm{C}$ & $01|02| 03|04| 07$ & 5 \\
\hline rs430600 & chr1 & $\mathrm{T}>\mathrm{A} / \mathrm{T}>\mathrm{C}$ & PKN2 & Coding sequence variant & $\mathrm{C}$ & $02|03| 04|05| 06$ & 5 \\
\hline rs6557210 & chr6 & $\mathrm{G}>\mathrm{A}$ & SYNE1 & Intron variant & $\mathrm{C}$ & 01102104105 & 4 \\
\hline rs 10455097 & chr6 & $\mathrm{A}>\mathrm{C}$ & CD109 & Coding sequence variant & $\mathrm{C}$ & 02103104106 & 4 \\
\hline rs2721939 & chr8 & $\mathrm{C}>\mathrm{T}$ & TRPS1 & Intron variant & $\mathrm{C}$ & 01105107 & 3 \\
\hline rs6904364 & chr6 & $\mathrm{T}>\mathrm{C}$ & RMND1 & Intron variant & $\mathrm{C}$ & 05106107 & 3 \\
\hline rs2293889 & chr8 & $\mathrm{T}>\mathrm{C} / \mathrm{T}>\mathrm{G}$ & TRPS1 & Intron variant & $\mathrm{C}$ & 01105 & 2 \\
\hline rs1529868 & $\operatorname{chr} 2$ & $\mathrm{C}>\mathrm{T}$ & GREB1 & Intron variant & $\mathrm{C}$ & 05106 & 2 \\
\hline rs17082236 & chr6 & $\mathrm{C}>\mathrm{A}$ & SYNE1 & Coding sequence variant & $\mathrm{C}$ & 01 & 1 \\
\hline
\end{tabular}

Class 'A' is equal to 'high-associated', class ' $\mathrm{B}$ ' is equal to 'strong-associated' and class 'C' is equal to 'Associated' SNPs.

occurred within this family and were transmitted or imported from generation to generation. Moreover, a list of 'high-associated' and 'strong-associated' polymorphisms that are directly related to endometriosis were identified and classified in each one of the seven patients (Table II). All tests were run with the Demetra App using default parameters on the human reference genome GRCh38 and the human mitochondrial complete genome (NCBI: NC_012920.1). Furthermore, the Demetra App was also successfully evaluated with different well-reported cases of SNPs located in genes, which may play a critical role in the development of endometriosis, as shown in Table II. 


\section{Discussion}

Demetra App services aid the diagnosis of endometriosis using a patient's genetic profile through provided information that will eventually help to identify a patient's predisposition to endometriosis in the very early stages, even without any symptoms. In the case where medical experts lack a clear etiology for the patient's condition, Demetra App results can provide useful information about the patient profile and a list of the most critical polymorphisms present in the patient's genome and their association with several biological pathways.

The quality of the data for variants identified in the VCF file uploaded by the user many times may provide low reliability and pause several limitations. To deal with such issues, the Demetra App validates the VCF file and remove variants that did not pass the quality control thresholds. On the other hand, it can also enable the user to upload the raw sequences or genotype data and provides a pre-processed analysis through which a generated VCF file is passed into the main pipeline of the webserver. Thus, the user has the option to analyze both VCF and FASTA files without any restrictions.

DAD contains all the identified SNPs related to endometriosis, classified into three major classes. The quality of the information in the individual databases has possible limitations, and clinical databases may include nonverified annotations, as clinical research is being produced at ever faster rates. In an effort to ensure the predictive performance and the reliability of the system, so far, we opted for the manual update of the SNP DAD following validation and classification of the candidate SNPs by a team of medical experts.

In conclusion, endometriosis is an inherited multifactorial illness that is usually detected at a fairly advanced stage, preventing doctors from treating it well from an early stage. The Demetra App was designed to support physician diagnosis from the early stages by using the genomic data of the patient. The comprehensible interface of the Demetra App was designed to be used besides the clinical genomics scientists by many other health experts. Its output presents the examined patient's profile through which the user is provided with a structured set of results in various categories, which are generated based on the list of the most predictable candidate gene variants related to endometriosis. The majority of the current clinical genomics tools, web tools, and applications are scientifically oriented for geneticists and bioinformaticians and are not developed to be executed by medical doctors or other scientists. In this sense, the Demetra App is an easy-to-use integrated public web server for endometriosis, designed with the aim of bringing personalized medicine and personal genomics tools to the scientific community.

\section{Acknowledgements}

The authors would like to thank Dr Hans M. Albertsen of Juneau Biosciences (USA) for providing the test WES data from a previous study (29) and for providing critical view of the manuscript.

\section{Funding}

EE received funding by the project 'INSPIRED-The National Research Infrastructures on Integrated Structural
Biology, Drug Screening Efforts and Drug Target Functional Characterization' (Grant MIS 5002550) and by the project: 'OPENSCREENGR An Open-Access Research Infrastructure of Chemical Biology and Target-Based Screening Technologies for Human and Animal Health, Agriculture and the Environment' (Grant MIS 5002691), which are implemented under the Action 'Reinforcement of the Research and Innovation Infrastructure', funded by the Operational Program 'Competitiveness, Entrepreneurship and Innovation' (NSRF 2014-2020) and co-financed by Greece and the European Union (European Regional Development Fund).

\section{Availability of data and materials}

The datasets used and/or analyzed during the current study are available from the corresponding author on reasonable request.

\section{Authors' contributions}

LP, DV, GNG, IM, MM, MIZ, DAS and EE substantially contributed to the conception or design of the study, including the acquisition, analysis, or interpretation of the data for the study. LP, DV, GNG, IM, MM, MIZ, DAS and EE contributed towards drafting the study or revising it critically for important intellectual content and approved the version to be published. All authors agreed to be accountable for all aspects of the work in ensuring that questions related to the accuracy or integrity of any part of the work are appropriately investigated and resolved. All authors have read and approved the final manuscript. GNG and EE confirm the authenticity of the datasets used. EE, DV and LP confirm the origin of all data selected from public databases.

\section{Ethics approval and consent to participate}

The test WES data used were from a previous study (29), and thus no ethics approval was required for the present study, as this was previously obtained.

\section{Patient consent for publication}

Not applicable.

\section{Competing interests}

DAS is the Editor-in-Chief for the journal, but had no personal involvement in the reviewing process, or any influence in terms of adjudicating on the final decision, for this article. The other authors declare that they have no competing interests.

\section{References}

1. Halis G and Arici A: Endometriosis and inflammation in infertility. Ann N Y Acad Sci 1034: 300-315, 2004.

2. Zondervan KT, Becker CM, Koga K, Missmer SA, Taylor RN and Viganò P: Endometriosis. Nat Rev Dis Primers 4: 9, 2018.

3. Sapkota Y, Steinthorsdottir V, Morris AP, Fassbender A, Rahmioglu N, De Vivo I, Buring JE, Zhang F, Edwards TL, Jones S, et al; iPSYCH-SSI-Broad Group: Meta-analysis identifies five novel loci associated with endometriosis highlighting key genes involved in hormone metabolism. Nat Commun 8: 15539, 2017. 
4. Vassilopoulou L, Matalliotakis M, Zervou MI, Matalliotaki C, Krithinakis K, Matalliotakis I, Spandidos DA and Goulielmos GN: Defining the genetic profile of endometriosis. Exp Ther Med 17: 3267-3281, 2019.

5. Alborzi S, Hosseini-Nohadani A, Poordast T and Shomali Z: Surgical outcomes of laparoscopic endometriosis surgery: A 6 year experience. Curr Med Res Opin 33: 2229-2234, 2017.

6. Anastasiu CV, Moga MA, Elena Neculau A, Bălan A, Scârneciu I, Dragomir RM, Dull AM and Chicea LM: Biomarkers for the noninvasive diagnosis of endometriosis: state of the art and future perspectives. Int J Mol Sci 21: 21, 2020.

7. Goulielmos GN, Matalliotakis M, Matalliotaki C, Eliopoulos E, Matalliotakis I and Zervou MI: Endometriosis research in the -omics era. Gene 741: 144545, 2020.

8. Palmer SS and Barnhart KT: Biomarkers in reproductive medicine: The promise, and can it be fulfilled? Fertil Steril 99: 954-962, 2013

9. de Sanctis V, Matalliotakis M, Soliman AT, Elsefdy H, Di Maio S and Fiscina B: A focus on the distinctions and current evidence of endometriosis in adolescents. Best Pract Res Clin Obstet Gynaecol 51: 138-150, 2018.

10. Agarwal SK, Chapron C, Giudice LC, Laufer MR, Leyland N, Missmer SA, Singh SS and Taylor HS: Clinical diagnosis of endometriosis: a call to action. Am J Obstet Gynecol 220: 354 e1-354.e12, 2019

11. Tam V, Patel N, Turcotte M, Bossé Y, Paré G and Meyre D Benefits and limitations of genome-wide association studies. Nat Rev Genet 20: 467-484, 2019.

12. Khan R and Mittelman D: Consumer genomics will change your life, whether you get tested or not. Genome Biol 19: 120, 2018.

13. Roberts J and Middleton A: Genetics in the 21st Century: Implications for patients, consumers and citizens. F1000 Res 6 : 2020, 2017.

14. Perakakis N, Yazdani A, Karniadakis GE and Mantzoros C: Omics, big data and machine learning as tools to propel understanding of biological mechanisms and to discover novel diagnostics and therapeutics. Metabolism 87: A1-A9, 2018.

15. Yang J, Li Y, Liu Q, Li L, Feng A, Wang T, Zheng S, Xu A and Lyu J: Brief introduction of medical database and data mining technology in big data era. J Evid Based Med 13: 57-69, 2020.

16. Xu J, Kim S, Song M, Jeong M, Kim D, Kang J, Rousseau JF, Li X, Xu W, Torvik VI, et al: Building a PubMed knowledge graph. Sci Data 7: 205, 2020.

17. Liu JL and Zhao M: A PubMed-wide study of endometriosis Genomics 108: 151-157, 2016.

18. Allot A, Peng Y, Wei CH, Lee K, Phan L and Lu Z: LitVar: A semantic search engine for linking genomic variant data in PubMed and PMC. Nucleic Acids Res 46 (W1): W530-W536, 2018.
19. Sherry ST, Ward MH, Kholodov M, Baker J, Phan L, Smigielski EM and Sirotkin K: dbSNP: The NCBI database of genetic variation. Nucleic Acids Res 29: 308-311, 2001.

20. Brown GR, Hem V, Katz KS, Ovetsky M, Wallin C, Ermolaeva O, Tolstoy I, Tatusova T, Pruitt KD, Maglott DR, et al: Gene: A gene-centered information resource at NCBI. Nucleic Acids Res 43 (D1): D36-D42, 2015.

21. Kim S, Yeganova L, Comeau DC, Wilbur WJ and Lu Z: PubMed Phrases, an open set of coherent phrases for searching biomedical literature. Sci Data 5: 180104, 2018.

22. Hamosh A, Scott AF, Amberger JS, Bocchini CA and McKusick VA: Online Mendelian Inheritance in Man (OMIM), a knowledgebase of human genes and genetic disorders. Nucleic Acids Res 33: D514-D517, 2005.

23. Joseph S and Mahale SD: Endometriosis Knowledgebase: a gene-based resource on endometriosis. Database (Oxford) 2019: baz062, 2019

24. Landrum MJ, Lee JM, Benson M, Brown GR, Chao C, Chitipiralla S, Gu B, Hart J, Hoffman D, Jang W, et al: ClinVar: Improving access to variant interpretations and supporting evidence. Nucleic Acids Res 46 (D1): D1062-D1067, 2018.

25. Danecek P, Auton A, Abecasis G, Albers CA, Banks E, DePristo MA, Handsaker RE, Lunter G, Marth GT, Sherry ST, et al; 1000 Genomes Project Analysis Group: The variant call format and VCFtools. Bioinformatics 27: 2156-2158, 2011.

26. Banchs RE: Text Mining With MATLAB. Springer, New York, NY, 2013.

27. Xiao H, Yang L, Liu J, Jiao Y, Lu L and Zhao H: Protein-protein interaction analysis to identify biomarker networks for endometriosis. Exp Ther Med 14: 4647-4654, 2017.

28. Jurca G, Addam O, Aksac A, Gao S, Özyer T, Demetrick D and Alhajj R: Integrating text mining, data mining, and network analysis for identifying genetic breast cancer trends. BMC Res Notes 9: 236, 2016

29. Albertsen HM, Matalliotaki C, Matalliotakis M, Zervou MI, Matalliotakis I, Spandidos DA, Chettier R, Ward K and Goulielmos GN: Whole exome sequencing identifies hemizygous deletions in the UGT2B28 and USP17L2 genes in a three generation family with endometriosis. Mol Med Rep 19: 1716-1720, 2019.

This work is licensed under a Creative Commons Attribution-NonCommercial-NoDerivatives 4.0 International (CC BY-NC-ND 4.0) License. 